\title{
Hypomania in clinical practice
}

\author{
Daniel J. Smith \& S. Nassir Ghaemi
}

Abstract Recent work suggests that a broad clinical spectrum of bipolar disorder is more common than previously thought and that the disorder may affect up to $5 \%$ of the population. The correct definition and diagnosis of hypomania is central to the identification of bipolar disorder. In this review we focus on recent diagnostic and clinical advances relating to bipolar disorder, with particular reference to hypomanic states. We also highlight some of the controversies in this field and discuss ways in which clinicians might improve their detection of bipolar disorders.

'When you're high it's tremendous. The ideas and feelings are fast and frequent... Shyness goes, the right words and gestures are suddenly there, the power to captivate others a felt certainty ... Sensuality is pervasive and the desire to seduce and be seduced irresistible' (Jamison, 1995: p. 67).

'In hypomania, there is an increased pressure of speech with prolixity, an abnormal liveliness of expressive movements, superficial bustling activity and a tendency to be argumentative and irritable if thwarted in any way. The patient is interested in everything, starts many projects, and finishes none... the elated mood leads to faulty judgement and a lack of consideration for others ... hypersexuality may lead to venereal disease in men and pregnancy in women ... the mental illness may be obvious only to the relatives and doctors, and other outside observers may merely regard the patient as a cheerful chap or somewhat of a "card"' (Fish, quoted by Hamilton, 1974: p. 73).

Although Kay Jamison's evocative description of the early stages of mania contrasts with the phenomenological account provided by Frank Fish (writing in the 1960s), these two quotations illustrate a fundamental point about the presentation and diagnosis of hypomanic states. People with mild manic symptoms rarely complain about feeling unwell (indeed, many enjoy their 'supernormal' levels of functioning), whereas family members and psychiatrists will usually be quick to recognise the behavioural manifestations of the illness. In this review, we focus on some of the recent advances in our understanding of the presentation and detection of hypomania.

\section{Diagnostic issues}

The study of less severe forms of bipolar disorder has been neglected for many years, but developments in clinical phenomenology, nosology and epidemiology have given rise to renewed interest in the correct definition and diagnosis of hypomania.

\section{Categories v. dimensions}

Many of the diagnoses used in psychiatry, including those described in ICD-10 (World Health Organization, 1992) and DSM-IV (American Psychiatric Association, 1994), are categorical in nature and based on a checklist approach that specifies an arbitrary number of symptoms as a threshold for diagnosis. Although these classifications have been of considerable value, they fail to reflect the clinical reality that psychiatric presentations are often dimensional rather than allor-nothing phenomena.

For mood disorders, one of the consequences of this has been the proliferation of a large collection of diagnoses, ranging from major (unipolar) depression through to severe psychotic disorders such as schizoaffective disorder. Not surprisingly, the dividing line between what might be considered normal temperament and full-blown mood disorder can often appear blurred. A good illustration of this is the retention of sub-threshold disorders such as dysthymia and cyclothymia in ICD-10 and DSM-IV. For bipolar disorder, the accurate detection of hypomania is of pivotal diagnostic

\footnotetext{
Daniel Smith is a clinical lecturer in the Department of Psychological Medicine at Cardiff University (Henry Wellcome Building for Biomedical Research, Academic Avenue, Heath Park, Cardiff CF14 4XN, UK. E-mail: djsmith1999@hotmail.com). He has research interests in the classification and genetics of bipolar disorder. Nassir Ghaemi is Associate Professor of Psychiatry and Public Health and Director of the Bipolar Disorders Research Program at Emory University, in the US state of Georgia. He has published widely on nosological and treatment aspects of bipolar disorder.
} 
importance and has considerable implications for treatment.

\section{What is the correct definition of hypomania?}

Although ICD-10 and DSM-IV are generally in agreement over what constitutes a core manic symptom, there are important differences in the guidance that they provide for the diagnosis of hypomania. In ICD-10, hypomania is considered to be a lesser degree of mania lasting 'several days' and causing 'considerable interference with work or social activity'. In contrast, DSM-IV stipulates that hypomania occurs 'without [my italics] marked social or occupational dysfunction' (leading to a diagnosis of bipolar II disorder). Full-blown mania in DSM-IV refers to any persistent elevation of mood lasting more than 1 week that causes functional impairment (and leads to a diagnosis of bipolar I disorder).

Thus, many patients with ICD-10-defined hypomania may actually satisfy DSM-IV criteria for mania. This confusion is compounded in the UK by an apparent overuse of the term 'hypomania'. It is not uncommon for a patient who has been hospitalised for weeks with severe mania to be given a discharge diagnosis of hypomania (Goodwin, 2002).

Some of the core manic symptoms of hypomania may be more common than others. For example, 'increased activity' and 'increased energy' are almost universal, whereas 'increased talkativeness' does not feature in over a quarter of hypomanic presentations (Fig. 1) (Wicki \& Ansgt, 1991).

Prevalence rates for bipolar disorders depend on the diagnostic criteria applied to people with recurrent major depression, and central to this issue is the correct definition of the boundaries of hypomania. The minimum duration required for a diagnosis of hypomania in the DSM has changed significantly over the years. In the Research Diagnostic Criteria it was 2 days (Spitzer et al, 1978), in DSM-III (1980) and DSM-III-R (1987) it was not specified, and in DSM-IV it has been set arbitrarily at 4 days. Within the past few years, a group of experts on bipolar disorder have recommended reverting to the threshold of 2 days, for two reasons. First, most people with bipolar disorder report a median duration of hypomanic symptoms of 1-3 days (rather than 4 or more) (Benazzi \& Akiskal, 2003). Second, several longitudinal studies have reported that people who experience brief hypomanic periods do not differ from those with longer periods in terms of features age at illness onset, family history of mania and lifetime illness chronicity (Akiskal et al, 2000; Angst et al, 2003; Benazzi \& Akiskal, 2003; Judd et al, 2003).

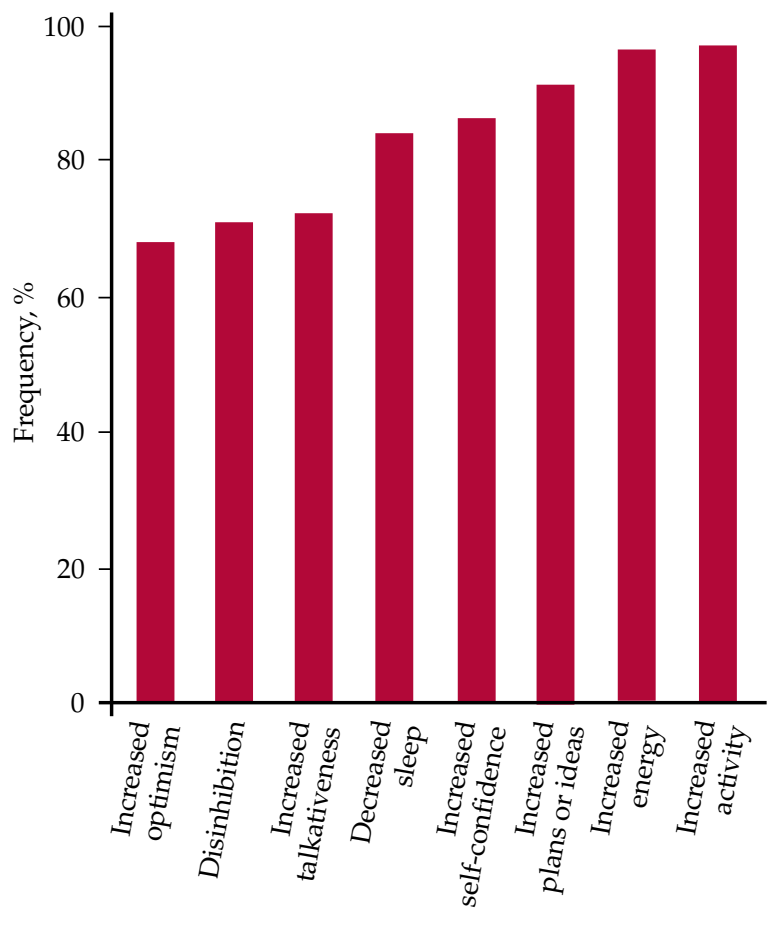

Fig. 1 Frequency of core manic symptoms in hypomania (data from Wicki \& Angst, 1991).

\section{Dysphoric (or mixed) hypomania}

The traditional view of hypomania as a predominantly euphoric mental state is challenged by recent studies. Many people with bipolar disorder experience 'dysphoric' or 'mixed' hypomanic periods that can be diagnostically challenging (Akiskal et al, 2003a). Some studies have estimated that at least half of all mood episodes in bipolar disorder are mixed presentations (Cassidy \& Carroll, 2001).

In a recent 7-year naturalistic study of 908 people with bipolar disorder within the Stanley Bipolar Treatment Network, Suppes et al (2005) found that out of 1044 visits in which the patients met DSM-IV criteria for hypomania, in $57 \%$ of cases they also met criteria for 'mixed hypomania' (defined as a Young Mania Rating Scale score $\geq 12$ and an Inventory of Depressive Symptomatology score $\geq 15$ ). Mixed hypomania was much more likely in women than in men, supporting previous findigns of an overrepresentation of mixed states in female patients with bipolar disorder (Arnold et al, 2000; Benazzi, 2003).

\section{Differential diagnosis of hypomania}

The diagnosis of hypomania can also be more difficult because hypomanic symptoms can feature prominently in a number of other conditions. A commonly encountered difficulty is the 


\section{Box 1 Differential diagnosis of hypomania}

- Levels of elevated mood and overactivity that lie within normal limits

- Drug misuse: cannabis, amphetamine, methylenedioxymethamphetamine (MDMA, ecstasy) and cocaine

- Cyclothymia

- Cluster B personality disorder (e.g. borderline personality disorder)

- Attention-deficit hyperactivity disorder

- Endocrinopathy such as hyperthyroidism (rare)

differentiation of hypomania from periods of elevated mood, overactivity and grandiosity that can occur in individuals with extrovert or cyclothymic personalities. Similarly, people with DSM-IV cluster B personality disorders (particularly borderline personality disorder) who do not have a mood disorder can present with high levels of affective arousal that can mimic hypomania. Diagnosis in these patients is often made even more difficult by their concurrent use of drugs such as cannabis, amphetamine and cocaine.

Leaving aside concerns about the clinical validity of a diagnosis of adult attention-deficit hyperactivity disorder (ADHD) (Asherson, 2004; Zwi \& York, 2004), some individuals presenting with hyperactivity, disinhibition and inattention may be misdiagnosed as being hypomanic. However, the differentiation of ADHD from hypomania should usually be straightforward if based on a systematic assessment for other hypomanic features such as elevated or irritable mood, inflated self-esteem and decreased need for sleep.

As with all psychiatric diagnoses, care should be taken to eliminate a clear organic cause for hypomanic symptoms, such as the use of stimulant drugs or an endocrine abnormality like hyperthyroidism. Box 1 shows the most common differential diagnoses for hypomania. A key issue in its differential diagnosis is that the psychiatric assessment should focus not only on the present mental state but also on the longitudinal history and temporal pattern of recurrent mood episodes (be they depressive, manic or mixed).

\section{The underdiagnosis of bipolar disorder}

A large body of evidence has now accumulated to support the assertion that bipolar disorder is underrecognised in everyday clinical practice. When the duration criterion for hypomania is reduced from
4 to 2 days, the prevalence of bipolar disorder rises from 1\% to at least 5\% (Angst, 1998; Akiskal et al, 2000). This rise occurs at the expense of recurrent unipolar depression, suggesting that between 25\% and $50 \%$ of all people with recurrent depression may in fact be part of a bipolar group (Hantouche et al, 1998; Angst \& Gamma, 2002; Benazzi \& Akiskal, 2003).

Using the Zurich cohort of 4547 young adults, who were formally assessed six times between 1978 and 1999, Angst et al (2003) tested the diagnostic criteria of DSM-IV hypomania and attempted to develop and validate criteria for softer expressions of bipolar II disorder. They used the standard DSM-IV definition of hypomania in addition to 'Zurich strict' and 'Zurich broad' criteria (Box 2). They found the prevalence of bipolar II disorder to be $2 \%$ under DSM-IV criteria, $5 \%$ under Zurich strict criteria and $11 \%$ under Zurich broad criteria. Interestingly, the clinical validity of diagnoses using both sets of Zurich criteria was supported by data on family history, illness course and clinical characteristics. Under the Zurich broad criteria, up to half of those with recurrent major depression in this cohort could be considered to have bipolar II disorder.

Several additional reports support a higher prevalence and clinically significant spectrum of bipolar disorders (Angst, 1998; Akiskal et al, 2000; Judd et al, 2003). For example, when people with a diagnosis of recurrent major depression are systematically assessed for a past history of hypomanic symptoms, $45 \%$ satisfy the diagnostic criteria for a bipolar disorder, suggesting that the detection of hypomania in everyday practice is

\section{Box 2 The Zurich criteria for hypomania}

Strict criteria

A Euphoria, irritability or overactivity

B At least three of the seven DSM-IV symptoms of hypomania

C Hypomanic symptoms of at least 1 day's duration

D Experience of negative consequences of hypomanic periods

\section{Broad criteria}

A Euphoria, irritability or overactivity

B At least two of the seven DSM-IV symptoms of hypomania

C No minimum duration of hypomanic symptoms

D No requirement to have experienced negative consequences of hypomania

(Angst et al, 2003, with permission) 
deficient, even when using criteria that are overly restrictive (Allilaire et al, 2001).

Although the concept of a broadened bipolar spectrum has gained some prominence in recent years, it must be noted that this area remains controversial. Baldessarini (2000) has argued that classic bipolar disorder is probably as close to a 'disease' as we have in modern psychiatry and that widespread acceptance of broadened definitions of bipolarity run the risk of trivialising the core concept. This view has important implications for biological research into bipolar (and unipolar) disorder, including neuropsychological, imaging and genetic studies. It would also affect the future design of treatment trials for depression and bipolar disorder. If one accepts that a proportion of people with recurrent depression may indeed have a bipolar disorder (even if broadly defined), this raises the uncomfortable possibility that much of the data on the efficacy of antidepressants in unipolar depression may have been derived from clinical populations that contain a significant proportion of people with bipolar depression.

\section{Why is hypomania under- diagnosed?}

Several factors contribute to the underdiagnosis of hypomania in clinical practice.

\section{Depression dominates the natural history of bipolar II disorder}

Bipolar disorder is characterised by oscillations between depression and hypomania and/or mania. For many years it was believed that mood symptoms and levels of functioning returned to normal between episodes of illness, but recent work has challenged this idea. Long-term follow-up studies of the symptomatic status of people with bipolar disorder have demonstrated that clinically relevant symptoms are extremely common and can have a major impact on psychosocial functioning.

Judd et al (2003) prospectively followed 86 individuals with bipolar II disorder for a mean duration of 13.4 years and found that depressive symptoms were reported in $50.3 \%$ of the weeks studied. Hypomanic symptoms were reported in only $3.6 \%$ of the weeks studied. In a similar study of 146 people with bipolar I disorder followed over 12.8 years, individuals were symptomatic for $47 \%$ of the study time (Judd et al, 2002). As with bipolar II disorder, depressive symptoms predominated over manic or hypomanic symptoms $(31.9 \% v$. $14.8 \%$ of total follow-up weeks) (Judd et al, 2002).
The clinical course of bipolar disorders is therefore dominated by depression rather than hypomania. This is especially true for bipolar II disorder, where the ratio of time spent with depressive symptoms relative to time with hypomanic symptoms is about 30:1 (Judd et al, 2003).

\section{People tend to seek help only for depressive episodes}

Traditionally, we have been trained to diagnose bipolar disorder on the basis of manic presentations against a background of recurrent depression rather than taking into account other, more subtle clinical variables. Unsurprisingly, most people with bipolar disorder do not seek help during hypomanic periods. As a result, both primary care physicians and psychiatrists usually come into contact with them only during depressive episodes. Furthermore, patients recollection of previous hypomanic symptoms at this time is often poor. Depressive cognitive distortions tend to make their recall of elevated periods less likely and some patients may consciously (and unconsciously) withhold information about hypomanic symptoms, for example because of a reluctance to take moodstabilising medication. Most clinicians are also much better at assessing depressive symptoms than hypomanic symptoms (Sprock, 1988).

The individual's lack of insight during hypomanic and manic episodes is a major factor in the underdetection of bipolar disorder. Although impaired insight is well documented as a core feature of psychosis, it has been much less studied in mood disorders. Insight has been shown to be significantly more impaired during hypomania and mania than during depression, and impaired insight appears to be almost as prominent a feature in mania as in psychosis (Ghaemi et al, 1995). A meta-analysis of studies found that impaired insight is a statedependent feature of mania and should probably, as with psychosis, form part of the diagnostic picture (Ghaemi \& Rosenquist, 2004).

For these reasons, it is essential that a corroborative history be obtained from a first-degree relative, partner or close friend during any assessment of possible bipolar disorder. Including family members in the assessment significantly increases the detection rate of bipolar disorder. In a study of the prodromal features of depression and mania, families reported behavioural manifestations of mania more than twice as frequently as patients $(47 \%$ v. 22\%) (Keitner et al, 1996). In our own clinical experience, we often observe a striking disparity between the account of hypomanic features provided by patients and by their close relatives. 
Although this kind of assessment may be timeconsuming, it can significantly alter the long-term prognosis for many patients. Clinicians' time can be saved by a brief informative telephone call to a family member rather than hours of inaccurate or evasive interviews with the patient. Involving family members in assessment also has a number of less obvious advantages. It may bring to light a previously unknown family history of bipolar disorder or psychosis and can help some patients to engage more fully in their treatment plans.

\section{Bipolar depression may differ from unipolar depression}

A more systematic search for previous symptoms of hypomania may be prompted by a clinical suspicion that a depressed patient is presenting with bipolar rather than unipolar depression. Although it is widely held that people with bipolar depression are indistinguishable on clinical grounds from those with unipolar depression, a number of recent studies challenge this idea.

In a 3-year prospective follow-up study of 206 consecutive out-patients with depression and no prior history of bipolar disorder, 41 (20\%) developed an episode of mania (Akiskal et al, 1983). Several variables were associated with bipolar outcome: depressive episodes of acute onset, frequent recurrence and brief duration; a family history of bipolar disorder; post-partum depression; psychomotor retardation or atypical features; and antidepressant-associated hypomania. The strongest associations with bipolar outcome were a family history of bipolar disorder and antidepressantassociated hypomania, with positive predictive values of $94 \%$ and $100 \%$ respectively (Akiskal et al, 1983).

In a small study of 27 individuals with unipolar depression and 27 age- and gender-matched people with bipolar depression, Mitchell et al (1992) found that those with bipolar depression tended to exhibit less psychomotor slowing, more agitation, more atypical symptoms and had shorter depressive episodes. Atypical depressive features (including mood reactivity, overeating, oversleeping, excessive fatigue, so-called leaden paralysis in the limbs and sensitivity to interpersonal rejection) appear to be at least twice as common in bipolar as in unipolar depression (Angst et al, 2005).

A comparison of 39 depressed patients with bipolar I disorder with age- and gender-matched depressed patients with unipolar depression found that the former were significantly more likely to have experienced an episode of psychotic depression in the past (Mitchell et al, 2001). Goldberg et al (2001)

\section{Box 3 Indicators of bipolarity in apparently} unipolar depression

- First-degree relative with bipolar disorder

- Antidepressant-induced mania or hypomania

- Multiple family members with major depression

- Early age at onset of depression (less than 25 years old)

- Psychotic features (before age 35)

- Atypical features

- Post-partum illness

- Brief recurrent episodes of depression (less than 6 months' duration)

conducted a 15-year follow-up of 74 young adults hospitalised for severe unipolar depression and found that $27 \%$ subsequently developed hypomania, with an additional 19\% experiencing at least one episode of full-blown mania. The presence of mood-congruent psychotic symptoms during the index depressive episode was strongly predictive of bipolar disorder, with 8 out of 10 patients with psychotic depression eventually becoming bipolar during the 15-year follow-up period.

A number of other variables have been associated with bipolar outcome in those with apparent unipolar disorder, although the evidence for these is weaker. These include hyperthymic personality, which in some studies (Akiskal et al, 2000) but not in others (Cassano et al, 1999) has been associated with a family history of bipolar disorder. Studies of the natural history of mood disorders suggest that bipolar depressive episodes tend to be shorter than unipolar depressive episodes (with a median duration of 3-6 months compared with 6-12 months) (Goodwin \& Jamison, 1990). Acute but not prophylactic response to antidepressants (often referred to as 'antidepressant wear-off') and a history of non-response to multiple courses of antidepressants might also be predictive of bipolar outcome (Goodwin \& Jamison, 1990). Possible indicators of bipolarity in apparently unipolar depression are listed in Box 3.

\section{The bipolar nature of depressive mixed states}

As noted above, the apparent 'bipolarity' of mood episodes in bipolar disorder (a clear manic pole v. a clear depressive pole) may be a reflection of different proportions of manic and depressive symptoms within any given episode, rather than 
the occurrence of discrete 'pure manic' and 'pure depressive' episodes. Just as depressive symptoms occur during hypomanic periods of bipolar disorder, so hypomanic symptoms seem to occur during depressive episodes of the disorder.

In a cross-sectional analysis of both depressive and manic symptoms in a treated sample of 441 people with bipolar disorder, Bauer et al (2005) found that $68(15.4 \%)$ met DSM-IV criteria for a current hypomanic or manic episode and that most of these $(64 / 68,94.1 \%)$ also had clinically significant depressive symptoms.

This idea that mood episodes in bipolar disorder can often present as admixtures of depressive and hypomanic symptoms is supported by evidence that mixed depression may represent a clinical marker for bipolar disorder. In a consecutive sample of 226 individuals with bipolar II disorder and 151 with unipolar depression, Akiskal \& Benazzi (2003) assessed the prevalence of mixed depression during an index depressive episode. They defined mixed depression as DSM-IV major depression plus three or more concurrent hypomanic symptoms. Mixed depression was present in $58.4 \%$ of those in the bipolar II group and in $23.1 \%$ of those in the unipolar group. Those with mixed depression in the two groups shared similar clinical variables such as age at illness onset, atypical features and family history of bipolar disorder, suggesting that they might have a bipolar-spectrum disorder.

\section{The status of antidepressant- associated hypomania}

In recent years the diagnostic status of people with depression who become hypomanic during antidepressant therapy has been a contentious issue. Many psychiatrists believe that in patients with no previous history of hypomania, brief periods of hypomania occurring within weeks of starting antidepressants should not necessarily lead to a diagnosis of bipolar illness. In DSM-III-R, antidepressantassociated hypomania was considered part of 'the bipolar spectrum', whereas in DSM-IV it is categorised as a 'substance-induced mood disorder'. However, a consensus is re-emerging that people with depression who experience antidepressantassociated hypomania are truly bipolar.

This is a difficult area because many of the original descriptions of antidepressant-induced hypomania did not distinguish between patients with preexisting diagnoses of unipolar or bipolar disorder. In addition, most treatment trials of antidepressants for depression do not systematically assess patients for hypomanic symptoms during the recovery period. To address these deficiencies, Chun \&
Dunner (2004) reviewed 98 trials of antidepressants for depression where a systematic effort was made to separate participants with bipolar or unipolar disorder. Most of these studies (89\%) reported no cases of antidepressant-associated hypomania or mania, suggesting that antidepressants do not make individuals with correctly diagnosed unipolar disorder bipolar. In other words, when hypomania occurs as an adverse event following antidepressant therapy, it is because the patient has bipolar disorder. This is supported by evidence that individuals with unipolar depression who have antidepressantassociated hypomania and individuals with bipolar disorder who experience spontaneous hypomanias have very similar rates of mania in their family histories (Akiskal et al, 2003b).

The issue of whether antidepressants are helpful in both the short and long term in the treatment of bipolar depression is unresolved. In a recent metaanalysis of the short-term outcome of antidepressant treatment for bipolar depression, Gijsman et al (2004) found that antidepressants were effective in resolving depressive symptoms and did not appear to lead to more manic switches than did placebo. Conversely, in a literature review of hypomania or mania associated with recent antidepressant use in bipolar disorder, Goldberg \& Truman (2003) concluded that between $20 \%$ and $40 \%$ of all people with bipolar disorder were inherently susceptible to antidepressant-induced manias. They found this to apply to all classes of antidepressant, although selective serotonin reuptake inhibitors (SSRIs) may be less likely to cause hypomania or mania in bipolar disorder than are tricyclics or dual-action antidepressants such as venlafaxine.

It is likely that bipolar disorder represents a collection of heterogeneous disorders, some of which may be more sensitive to antidepressant-induced switching than others (Wehr et al, 1988). It also seems likely that a minority of people with bipolar disorder will require longer-term antidepressant therapy in combination with mood stabilisers to protect against depressive relapse (Altshuler et al, 2003).

Goldberg \& Truman (2003) have suggested that factors such as a strong genetic loading for bipolar illness, an early onset of illness, a history of multiple previous antidepressant trials and a history of antidepressant-induced manias should signal caution in the use of antidepressants for bipolar depression.

\section{Comorbidity}

Psychiatric comorbidity in bipolar disorder is extremely common and exerts a significant influence on the presentation, course and outcome. Comorbid 
conditions such as drug and alcohol misuse can mask the presentation of bipolar disorder, especially in the early stages of illness.

In the USA, McElroy et al (2001) evaluated 288 out-patients with bipolar disorder and found that $187(65 \%)$ also met DSM-IV criteria for at least one comorbid lifetime Axis I disorder, the most common being substance misuse $(n=78,42 \%)$. Alcohol misuse was present in 96 individuals (33\% of the total sample). There were also significant levels of marijuana (16\%), cocaine $(9 \%)$, stimulant drug $(9 \%)$, sedative drug ( $8 \%$ ) and opiate (7\%) misuse. Anxiety disorders were very common: $20 \%$ of the sample had a lifetime history of panic disorder; $16 \%$ social phobia; $9 \%$ obsessive-compulsive disorder; and $6 \%$ had a history of eating disorder. Current Axis I comorbidity was associated with an earlier age at onset of affective symptoms and the development of both cycle acceleration and more severe episodes over time.

DSM-IV Axis II comorbidity is also common in bipolar disorder and may exert an even greater influence on misdiagnosis and prognosis. Around one-third of people with bipolar disorder have a comorbid diagnosis of at least one personality disorder, and by far the most common of these is borderline personality disorder (Vieta \& Colom, 1999; Benazzi, 2000; Joyce et al, 2004). As many clinicians will recognise, mood episodes and affective instability are extremely common in borderline personality disorder. When individuals with borderline personality disorder are systematically assessed for the presence of bipolar features, rates of previously undiagnosed bipolarity of up to $69 \%$ have been reported (Deltito et al, 2001).

Although the relationship between bipolarity and borderline pathology is undoubtedly complex (for a review of this area see Paris, 2004, and Smith et al, 2004), many people with a diagnosis of borderline personality disorder may in fact have an undiagnosed bipolar-spectrum disorder and, as a result, may be denied effective treatments for their mood disorder. Conversely, some patients diagnosed with bipolar disorder will inevitably be more appropriately managed as patients with a borderline personality disorder (Bolton \& Gunderson, 1996).

\section{Improving the detection of hypomania in clinical practice}

We have already mentioned a number of straightforward measures for improving the detection of bipolar disorder (Box 4). Within the past few years, several assessment instruments have been developed to aid the detection of hypomanic symptoms.
Box 4 Simple ways of improving the detection of bipolar disorder

- Involving a reliable witness in the assessment of the patient

- Having a greater awareness of the natural history of bipolar disorder

- Recognising clinical features of bipolar depression and mixed affective states

- Having a greater awareness of subtle hypomanic symptoms and the impact of comorbidity

- Recognising the importance of bipolar disorder in a first-degree relative

- Recognising the likely bipolar status of antidepressant-associated hypomania

\section{Diagnostic criteria for bipolar-spectrum disorder}

One of us (S.N.G.) with colleagues has suggested structured diagnostic criteria for bipolar-spectrum disorder that describe patients who do not meet the strict DSM-IV criteria for bipolar disorder but who none the less have a number of important bipolar features (Ghaemi et al, 2001). These criteria, shown in Box 5 , give priority to a first-degree family history of bipolar disorder and a personal history of antidepressant-associated hypomania (Ghaemi et al, 2004). They also suggest that clustering of more subtle bipolar features may be used to aid diagnosis.

\section{The Mood Disorder Questionnaire}

The Mood Disorder Questionnaire (MDQ) is perhaps the best known screening instrument for bipolar disorder (Hirschfeld et al, 2000; available to patients at http://www.bipolar.com/mdq.htm). It is a brief form filled in by patients that can be easily incorporated into routine practice. In psychiatric out-patient settings, a positive response to 7 of the 13 core manic symptom questions (all occurring at the same time and associated with at least 'minor' functional impairment) gives a sensitivity for bipolar disorder of 0.73 and a specificity of 0.90 (Hirschfeld et al, 2000).

However, in general population settings this instrument has a much lower sensitivity, of only 0.28 , and a specificity of 0.97 (Hirschfeld et al, 2003). Miller et al (2002) tested the sensitivity of the MDQ in a population of individuals with bipolar disorder and found that it was much better at detecting bipolar I disorder (with a sensitivity of 0.70 ) than bipolar II disorder (sensitivity 0.30). Despite this, in 


\section{Box 5 Proposed diagnostic criteria for bipolar-} spectrum disorder

A At least one major depressive episode

B No spontaneous DSM-IV hypomanic or manic episodes

C Either of the following plus two from D, or both of the following plus one from $\mathrm{D}$ :

i first-degree relative with bipolar disorder

ii antidepressant-associated mania or hypomania

D If none from C, at least six of the following:

i hyperthymic personality

ii more than three depressive episodes

iii brief major depressive episodes (of less than 3 months' duration)

iv atypical depressive symptoms

$\mathrm{v}$ psychotic major depressive episodes

vi early age at onset (less than 25 years old)

vii post-partum depression

viii antidepressant wear-off (acute but not prophylactic response)

ix lack of response to more than two antidepressant trials

(From Ghaemi et al, 2001, with permission)

the clinical setting, many psychiatrists have found the MDQ to be useful in the overall assessment of bipolar features.

\section{The Bipolar Spectrum Diagnostic Scale}

The Bipolar Spectrum Diagnostic Scale (BSDS) is a narrative-based scale that has been developed to target symptoms of bipolar II disorder (Ghaemi $e t$ $a l, 2005)$. As it is a relatively little-known instrument we show it in Box 6 . With a team of colleagues one of us (S.N.G.) compared the sensitivity and specificity of this scale with those of the MDQ for 73 people with bipolar disorder and 20 with unipolar depression (Ghaemi et al, 2005). We found the MDQ to be better at correctly detecting bipolar I than bipolar II disorder (confirming Miller et al's findings mentioned earlier), whereas the BSDS was just as good at identifying both bipolar I and bipolar II disorder. This suggests that the BSDS may be particularly useful at detecting the broader spectrum of bipolar disorders.

When using instruments such as the MDQ and BSDS, due consideration should be given to clinical context. These screening tools should obviously be used to complement, rather than replace, careful clinical assessment.

\section{Conclusions}

We have tried to provide a useful summary of recent advances in the clinical presentation and diagnosis of hypomania. This area is not simply of academic interest but has implications for the way in which we assess and treat patients with recurrent mood disorders. Correctly identifying hypomania (using universally accepted criteria) will also influence many strands of mood disorder research, from animal models and genetics through to neuroimaging studies and the design of treatment trials of new medications.

\section{Declaration of interest}

S.N.G has been involved in the development and testing of the Bipolar Spectrum Diagnostic Scale.

\section{References}

Akiskal, H. S. \& Benazzi, F. (2003) Family history validation of the bipolar nature of depressive mixed states. Journal of Affective Disorders, 73, 113-122.

Akiskal, H. S., Walker, P., Puzantian, V. R., et al (1983) Bipolar outcome in the course of depressive illness: phenomenologic, familial, and pharmacologic predictors. Journal of Affective Disorders, 5, 115-128.

Akiskal, H. S., Bourgeois, M. L., Angst, J., et al (2000) Re-evaluating the prevalence of and diagnostic composition within the broad clinical spectrum of bipolar disorders. Journal of Affective Disorders, 59 (suppl. 1), 5s-30s.

Akiskal, H. S., Azorin, J. M. \& Hantouche, E. G. (2003a) Proposed multidimensional structure of mania: beyond the euphoricdysphoric dichotomy. Journal of Affective Disorders, 73, 7-18.

Akiskal, H. S., Hantouche, E. G., Allilaire, J.-F., et al (2003b) Validating antidepressant-associated hypomania (bipolar III): a systematic comparison with spontaneous hypomania (bipolar II). Journal of Affective Disorders, 73, 65-74.

Allilaire, J., Hantouche, E. G., Sechter, D., et al (2001) Frequency and clinical aspects of bipolar II disorder in a French multicentre study: EDIPEP. L'Encéphale, 27, 149-158.

Altshuler, L., Suppes, T., Black, D., et al (2003) Impact of antidepressant discontinuation after acute bipolar depression remission on rates of depressive relapse at 1-year follow-up. American Journal of Psychiatry, 160, 1252-1262.

American Psychiatric Association (1980) Diagnostic and Statistical Manual of Mental Disorders (3rd edn) (DSM-III). Washington, DC: APA.

American Psychiatric Association (1987) Diagnostic and Statistical Manual of Mental Disorders (3rd edn, revised) (DSM-III-R). Washington, DC: APA.

American Psychiatric Association (1994) Diagnostic and Statistical Manual of Mental Disorders (4th edn) (DSM-IV). Washington, DC: APA.

Angst, J. (1998) The emerging epidemiology of hypomania and bipolar II disorder. Journal of Affective Disorders, 50, 143-151.

Angst, J., \& Gamma, A. (2002) Prevalence of bipolar disorders: traditional and novel approaches. Clinical Approaches in Bipolar Disorders, 1, 10-14.

Angst, J., Gamma, A., Bennazzi, F., et al (2003) Toward a redefinition of subthreshold bipolarity: epidemiology and proposed criteria for bipolar-II, minor bipolar disorders and hypomania. Journal of Affective Disorders, 73, 133-146. 


\section{Box 6 The Bipolar Spectrum Diagnostic Scale (BSDS) (after Ghaemi et al, 2005, with permission)}

Instructions First, please read through the passages below, without filling in any blanks. Then read them again and put a tick in the box at the end of any sentences that you think fit you. Finally, tick one of the four boxes at the end of the passages.

Some individuals notice that their mood and/or energy levels shift drastically from time to time $\square$. They notice that their mood and/or energy levels are sometimes very low and at other times, very high $\square$.

During their low phases, these individuals often feel a lack of energy, a need to stay in bed or get extra sleep, and little or no motivation to do things they need to do $\square$. They often put on weight during these periods $\square$. During their low phases, these individuals often feel blue, sad all the time or depressed $\square$. Sometimes, they feel hopeless or even suicidal $\square$. Their ability to function at work or socially is impaired $\square$. Typically, these low phases last for a few weeks, but sometimes they last only a few days $\square$.

In between mood swings these individuals may experience a period of normal mood, during which their mood and energy level feel right and their ability to function is not disturbed $\square$.

They may then notice a marked shift or switch in the way they feel $\square$. They enter into a high period, when their energy increases above what is normal for them, and they often get many things done they would not ordinarily be able to do $\square$. During these high periods, these individuals sometimes feel as if they have too much energy or feel 'hyper' $\square$. Some feel irritable, on edge or aggressive $\square$. Some take on too many activities at once $\square$. Some spend money in ways that cause them trouble $\square$. They may be more talkative, outgoing or sexual during high periods $\square$. Sometimes, their behaviour during these high periods seems strange or annoying to others $\square$. Sometimes, these individuals get into difficulty with co-workers or the police at this time $\square$. Sometimes, they increase their alcohol or non-prescription drug use $\square$.

Now that you have read the passages and ticked the boxes, please tick one of the following four boxes

This story fits me very well, or almost perfectly

This story fits me fairly well

This story fits me to some degree, but not in most respects

This story does not really describe me at all

\section{Scoring the BSDS}

Each sentence ticked is worth 1 point. Add 6 points for 'fits me very well,' 4 points for 'fits me fairly well' and 2 points for 'fits me to some degree'.

\begin{tabular}{cl} 
Total score & Likelihood of bipolar disorder \\
\hline $0-6$ & Highly unlikely \\
$7-12$ & Low probability \\
$13-19$ & Moderate probability \\
$20-25$ & High probability
\end{tabular}

Optimum threshold for positive diagnosis: score of 13 or more.

Angst, J., Gamma, A., Benazzi, F., et al (2005) Atypical depressive syndromes in varying definitions. European Archives of Psychiatry and Clinical Neuroscience (Online First). DOI: 10.1007/ s00406-005-0600-z

Arnold, L. M., McElroy, S. \& Keck, P. E. Jr. (2000) The role of gender in mixed mania. Comprehensive Psychiatry, 41, 83-87.

Asherson, P. P. (2004) Bridging the service divide. Invited commentary on: Attention-deficit hyperactivity disorder in adults. Advances in Psychiatric Treatment, 10, 257-259.

Baldessarini, R. J. (2000) A plea for the integrity of the bipolar disorder concept. Bipolar Disorders, 2, 3-7.

Bauer, M. S., Simon, G. E., Ludman, E., et al (2005) 'Bipolarity' in bipolar disorder: distribution of manic and depressive symptoms in a treated population. British Journal of Psychiatry, 187, 87-88.
Benazzi, F. (2000) Borderline personality disorder and bipolar II disorder in private practice depressed outpatients. Comprehensive Psychiatry, 41, 106-110.

Benazzi, F. (2003) The role of gender in depressive mixed state. Psychopathology, 36, 213-217.

Benazzi, F. \& Akiskal, H. S. (2003) Refining the evaluation of bipolar II: beyond the strict SCID-CV guidelines for hypomania. Journal of Affective Disorders, 73, 33-38.

Bolton, S. \& Gunderson, J. G. (1996) Distinguishing borderline personality disorder from bipolar disorder: differential diagnosis and implications. American Journal of Psychiatry, 153, 1202-1207.

Cassano, G. B., Dell'Osso, L., Frank, E., et al (1999) The bipolar spectrum: a clinical reality in search of diagnostic criteria and 
an assessment methodology. Journal of Affective Disorders, 54, 319-328.

Cassidy, F. \& Carroll, B. J. (2001) The clinical epidemiology of pure and mixed manic episodes. Bipolar Disorders, 3, 35-40.

Chun, B. J. D. H. \& Dunner, D. L. (2004) A review of antidepressantinduced hypomania in major depression: suggestions for DSM-V. Bipolar Disorders, 6, 32-42.

Deltito, J., Martin, L., Riefkohl, J., et al (2001) Does borderline personality disorder belong to the bipolar spectrum? Journal of Affective Disorders, 67, 221-228.

Ghaemi, S. N. \& Rosenquist, K. J. (2004) Is insight in mania statedependent? A meta-analysis. Journal of Nervous and Mental Disease, 192, 771-775.

Ghaemi, S. N., Stoll, A. L. \& Pope, H. G. (1995) Lack of insight in bipolar disorder: the acute manic episode. Journal of Nervous and Mental Disease, 183, 464-467.

Ghaemi, S. N., Ko, J. K. \& Goodwin, F. K. (2001) The bipolar spectrum and the antidepressant view of the world. Journal of Psychiatric Practice, 7, 287-297.

Ghaemi, S. N., Hsu, D. J., Ko, J. Y., et al (2004) Bipolar spectrum disorder: a pilot study. Psychopathology, 37, 222-226.

Ghaemi, S. N., Miller, C. J., Berv, D. A., et al (2005) Sensitivity and specificity of a new bipolar spectrum diagnostic scale. Journal of Affective Disorders, 84, 273-277.

Gijsman, H. J., Geddes, J. R., Rendell, J. M., et al (2004) Antidepressants for bipolar depression: a systematic review of randomized, controlled trials. American Journal of Psychiatry, 161, 1537-1547.

Goldberg, J. F. \& Truman, C. J. (2003) Antidepressant-induced mania: an overview of current controversies. Bipolar Disorders, 5, 407-420.

Goldberg, J. F., Harrow, M. \& Whiteside, J. E. (2001) Risk for bipolar illness in patients initially hopitalised for unipolar depression. American Journal of Psychiatry, 158, 1265-1270.

Goodwin, G. (2002) Hypomania: what's in a name? British Journal of Psychiatry, 181, 94-95.

Goodwin, F. K. \& Jamison, K. R. (1990) Manic-Depressive Illness. New York: Oxford University Press.

Hamilton, M. (1974) Fish's Clinical Psychopathology. Bristol: John Wright and Sons.

Hantouche, E. G., Akiskal, H. S., Lancrenon, S., et al (1998) Systematic clinical methodology for validating bipolar-I disorder: data in mid-stream from a French national multi-site study (EDIPEP). Journal of Affective Disorders, 50, 163-173.

Hirschfeld, R. M. A., Williams, J. B. W., Spitzer, R. L., et al (2000) Development and validation of a screening instrument for bipolar spectrum disorder: the Mood Disorder Questionnaire. American Journal of Psychiatry, 157, 1873-1875.

Hirschfeld, R. M. A., Holzer, C. \& Calabrese, J. R. (2003) Validity of the Mood Disorder Questionaire: a general population study. American Journal of Psychiatry, 160, 178-180.

Jamison, K. R. (1995) An Unquiet Mind: A Memoir of Moods and Madness. New York: Knopf.

Joyce, P. R., Luty, S. E., McKenzie, J. M., et al (2004) Bipolar II disorder: personality and outcome in two clinical samples. Australian and New Zealand Journal of Psychiatry, 38, 433-438.

Judd, L. L., Akiskal, H. S., Schletter, P. J., et al (2002) The long-term natural history of the weekly symptomatic status of bipolar I disorder. Archives of General Psychiatry, 59, 530-537.

Judd, L. L., Akiskal, H. S., Schletter, P. J., et al (2003) A prospective investigation of the natural history of the long-term weekly symptomatic status of bipolar II disorder. Archives of General Psychiatry, 60, 261-269.

Keitner, G. I., Solomon, D. A., Ryan, C. E., et al (1996) Prodromal and residual symptoms in bipolar I disorder. Comprehensive Psychiatry, 37, 362-367.

McElroy, S. L., Altshuler, L. L., Suppes, T., et al (2001) Axis I psychiatric comorbidity and its relationship to historical illness variables in 288 patients with bipolar disorder. American Journal of Psychiatry, 158, 420-426.

Miller, C., Ghaemi, S. N., Klugman, J., et al (2002) Utility of mood disorder questionnaire and bipolar spectrum diagnostic scale. Program and Abstracts of the American Psychiatric Association 155th Annual Meeting. Philadelphia, PA: APA.

Mitchell, P., Parker, G., Jamison, K., et al (1992) Are there any differences between bipolar and unipolar melancholia? Journal of Affective Disorders, 25, 97-105.
Mitchell, P. B., Wilhelm, K., Parker, G., et al (2001) The clinical features of bipolar depression: a comparison with matched major depressive disorder patients. Journal of Clinical Psychiatry, 62, 212-216.

Paris, J. (2004) Borderline or bipolar? Distinguishing borderline personality disorder from bipolar spectrum disorders. Harvard Review of Psychiatry, 12, 140-145.

Smith, D. J., Muir, W. J. \& Blackwood, D. H. R. (2004) Is borderline personality disorder part of the bipolar spectrum? Harvard Review of Psychiatry, 12, 133-139.

Spitzer, R. L., Endicott, J. \& Robins, E. (1978) Research Diagnostic Criteria. Rationale and reliability. Archives of General Psychiatry, $35,773-782$.

Sprock, J. (1988) Classification of schizoaffective disorder. Comprehensive Psychiatry, 29, 55-71.

Suppes, T., Mintz, J., McElroy, S. M., et al (2005) Mixed hypomania in 908 patients with bipolar disorder evaluated prospectively in the Stanley Foundation Bipolar Treatment Network: a sex-specific phenomenon. Archives of General Psychiatry, 62, 1089-1096.

Vieta, E. \& Colom, F. (1999) Personality disorders in bipolar II patients. Journal of Nervous and Mental Disease, 187, 245-248.

Wehr, T. A., Sack, D. A., Rosenthal, N. E., et al (1988) Rapid cycling affective disorder: contributing factors and treatment responses in 51 patients. American Journal of Psychiatry, 145, 179-184.

Wicki, W. \& Angst, J. (1991) The Zurich Study, X. Hypomania in a 28- to 30-year old cohort. European Archives of Psychiatry and Clinical Neuroscience, 240, 339-348.

World Health Organization (1992) The ICD-10 Classification of Mental and Behavioural Disorders. Geneva: WHO.

Zwi, M. \& York, A. (2004) Attention-deficit hyperactivity disorder in adults: validity unknown. Advances in Psychiatric Treatment, 10, 248-256.

\section{MCQs}

1 Regarding hypomania and mania:

a manic episodes always last longer

b manic episodes can be associated with normal levels of social and occupational functioning

c hypomania can be regarded as a normal psychopathological state

d dysphoric hypomania is rare in bipolar disorder

e most hypomanic episodes in bipolar II disorder last at least 4 days.

2 Bipolar disorder may be underdiagnosed because:

a clinicians fail to ask about brief periods of elevated mood and activity

b clinicians do not consider brief periods of elevated mood to be diagnostically important

c many assessments of recurrent depression do not include taking a history from first-degree relatives or spouses

d people with bipolar disorder tend to present for help only when depressed

e the diagnosis of bipolar disorder has traditionally focused on the identification of mania rather than more subtle bipolar features of recurrent depression.

3 In comparing bipolar depression with unipolar depression:

a there are no significant phenomenological differences

b atypical symptoms are more common in unipolar depression

c where hypomania occurs as an adverse event in the treatment of unipolar depression, this probably means the patient has bipolar disorder 
d psychotic depression in earlier adulthood is strongly suggestive of bipolar disorder

e a family history of bipolar disorder is more common in bipolar depression.

4 The following may make the diagnosis of hypomania difficult:

a recent antidepressant therapy

b substance misuse

c borderline personality disorder

d symptoms of attention-deficit hyperactivity disorder

e thyrotoxicosis.

5 Methods to improve the correct detection of hypomania in clinical practice include:

a seeking accounts of behaviour from family members

b taking a comprehensive family history for mood disorders c recognising the potential diagnositic significance of antidepressant-associated hypomania

$\mathrm{d}$ probing for a past history of hypomanic symptoms in recurrent depression

e not using the MDQ or BSDS.

\section{MCQ answers}

$\begin{array}{lllllll}\text { 1 } & & \text { 2 } & & 3 & 4 & 5 \\ \text { a F } & \text { a T } & \text { a F } & \text { a T } & \text { a T } \\ \text { b F } & \text { b T } & \text { b F } & \text { b T } & \text { b T } \\ \text { c F } & \text { c T } & \text { c T } & \text { c T } & \text { c T } \\ \text { d F } & \text { d T } & \text { d T } & \text { d T } & \text { d T } \\ \text { e F } & \text { e T } & \text { e T } & \text { e T } & \text { e F }\end{array}$

